\title{
Alopecia Areata Incognita and Diffuse Alopecia Areata: Clinical, Trichoscopic, Histopathological, and Therapeutic Features of a 5-Year Study
}

\author{
Aurora Alessandrini ${ }^{1}$, Michela Starace ${ }^{1}$, Francesca Bruni ${ }^{1}$, Nicolò Brandi ${ }^{1}$, Carlotta Baraldi ${ }^{1}$, \\ Cosimo Misciali ${ }^{1}$, Pier Alessandro Fanti ${ }^{1}$, Bianca Maria Piraccini ${ }^{1}$
}

1 Department of Experimental, Diagnostic and Specialty Medicine, Division of Dermatology, University of Bologna, Bologna, Italy

Key words: alopecia areata incognita, diffuse alopecia areata, yellow dots, histopathology, therapy

Citation: Alessandrini A, Starace M, Bruni F, Brandi N, Baraldi C, Misciali C, Fanti PA, Piraccini BM. Alopecia areata incognita and diffuse alopecia areata: clinical, trichoscopic, histopathological, and therapeutic features of a 5-year study. Dermatol Pract Concept. 2019;9(4):272-277. DOI: https://doi.org/10.5826/dpc.0904a05

Accepted: July 23, 2019; Published: October 31, 2019

Copyright: @2019 Alessandrini et al. This is an open-access article distributed under the terms of the Creative Commons Attribution License, which permits unrestricted use, distribution, and reproduction in any medium, provided the original author and source are credited.

Funding: None.

Competing interests: The authors have no conflicts of interest to disclose.

Authorship: All authors have contributed significantly to this publication.

Corresponding author: Aurora Alessandrini, MD, Department of Experimental, Diagnostic and Specialty Medicine, Division of Dermatology, University of Bologna, Via Massarenti 1, 40138 Bologna, Italy. Email: aurora.alessandrini@alice.it

ABSTRACT Background: Alopecia areata is a nonscarring hair loss that usually causes round patches of baldness, but alopecia areata incognita (AAI) and diffuse alopecia areata (DAA) can cause a diffuse and acute pattern of hair loss.

Objective: To analyze the clinical, trichoscopic, histological, and therapeutic features of AAI and DAA.

Methods: The study was designed to include data of patients with histological diagnosis of AAI and DAA enrolled in our Hair Disease Outpatient Consultations.

Results: DAA had a greater involvement of the parietal and anterior-temporal regions, while AAI manifested itself mainly in the occipital-parietal regions. The most frequent pattern was empty yellow dots, yellow dots with vellus hairs, and small hair in regrowth, but the presence of pigtail hair was found almost exclusively in those with AAI. In cases of DDA, the finding of dystrophic hair and black dots was more frequent. The most frequent trichoscopic sign in both diseases was the presence of empty yellow dots, which, however, were described in a higher percentage in cases of DAA. The diseases have a benign course and are responsive to topical steroid therapy.

Conclusions: Trichoscopy is very important for the differential diagnosis between the 2 diseases and to select the best site for biopsy. In the presence of diffuse hair thinning, these entities must be considered. 


\section{Introduction}

Alopecia areata (AA) is a common cause of nonscarring hair loss that generally causes round patches of baldness on the scalp, or the whole scalp, or the entire body.

In some patients, however, AA is characterized by a diffuse hair loss, commonly misdiagnosed as telogen effluvium or androgenetic alopecia. The 2 most common variants of nonpatchy AA are alopecia areata incognita (AAI) and diffuse alopecia areata (DAA).

First described by Rebora in 1987 [1], AAI is more common (about $86 \%$ ) among young women, especially between 20 and 40 years old [2]. Clinically, it is characterized by widespread and severe hair loss, which develops rapidly in a few weeks in AAI, while DAA can appear over a prolonged period. Currently there are no studies that describe DAA and AAI in a standardized way; therefore, these clinical variants of AA are often not recognized.

The aim of our study is to analyze the clinical, trichoscopic, histological, and therapeutic features of these 2 entities and to underline main differences between them, especially through the aid of trichoscopy and skin biopsy, essential for diagnosis confirmation.

\section{Methods}

The study was designed to include data of patients with a histological diagnosis of AAI and DAA enrolled in our Hair Disease Outpatient Consultation, from April 2012 to April 2017.

Inclusion criteria were histological diagnosis of AAI or DAA and a signed informed consent. Exclusion criteria were diagnosis of other scalp diseases and/or unavailability of complete data. The study was approved by our University Ethics Committee.

General patient information was collected, including age at onset, clinical history of the disease, and previously prescribed therapies. Then each patient was subjected to global photography,

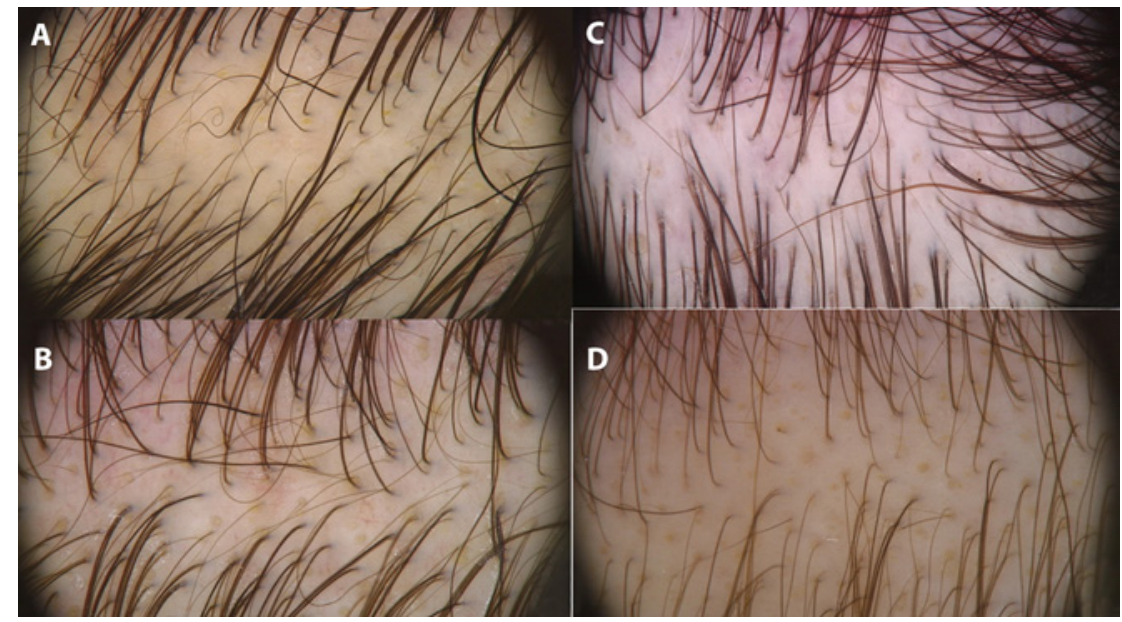

Figure 1. Trichoscopy (magnification $\times 20$ ) showing the most frequent patterns of alopecia areata incognita (A) with empty yellow dots, yellow dots with vellus hair, small hair in regrowth, and pigtail hair; (B) empty yellow dots, yellow dots with vellus hair, and small hair in regrowth. Trichoscopy (magnification $\times 20$ ) showing the most typical patterns of diffuse alopecia areata $(\mathrm{C})$ with empty yellow dots + yellow dots with vellus hair + dystrophic hair; (D) empty yellow dots + yellow dots with vellus hair + black dots + dystrophic hair. [Copyright: (C2019 Alessandrini et al.]

pull test, trichoscopy, and trichoscopy-guided scalp biopsy in a standardized technique using local anesthesia and a 5 -mm punch. Finally, the prescribed pharmacological treatment and its efficiency were reported.

\section{Results}

\section{Alopecia Areata Incognita}

Between April 2012 and April 2017, we diagnosed AAI in 107 patients, of whom 105 were female (98.13\%) and only 2 were male; most were Caucasians $(97.19 \%)$ and the average age was 40.55 years (range 21-79).

\section{Clinical Features}

All patients were characterized by increased and diffuse hair thinning without patches, lasting months, and were grouped in 3 degrees of severity as follows: 25 patients $(23.3 \%)$ with grade I, 33 patients $(30.8 \%)$ with grade II, and 49 patients $(45.7 \%)$ with grade III. A high percentage of patients were affected also by a concomitant androgenetic alopecia (102/107, 95.3\%). Pull test was positive in 32 patients $(29.9 \%)$, showing telogen roots. This clinical classification is based on the Ludwig scale [3].

\section{Trichoscopy}

Trichoscopy was performed in all patients and showed several patterns, especially in the parietal-occipital region (74.7\%) (Figure 1).

The different trichoscopic patterns were correlated with clinical presentation and degrees of severity: the most frequent pattern-empty yellow dots, yellow dots with vellus hair, small hair in regrowth, and pigtail hair-corresponded to grade III (49 patients); the pattern empty yellow dots, yellow dots with vellus hair, and small hair in regrowth was correlated with grade II (33 patients); and the remaining patterns were associated with grade I (25 patients) (Table 1).

\section{Pathology}

AAI is characterized by a preserved number of follicular units but a reduced number of terminal follicles, in particular those in anagen; in addition, a greater number of telogen units and numerous dilated infundibular hosts, rich in keratin and sebum, are evident. In 32 patients with positive pull test $(29.9 \%)$, a mild inflammatory infiltrate was found around the hair follicle in the upper dermis. The hair follicles in 
catagen and telogen and the telogenic germ units were instead increased. The main histopathological aspect, described in $100 \%$ of cases, was the presence of dilated infundibular ostia (0.02-0.05 $\mathrm{mm})$, empty or full of sebum and keratin, corresponding to yellow dots described by trichoscopy. Small follicles in telogen were found in 86 patients $(80.3 \%)$, mainly located at the bulge level. The number of vellus hair follicles and miniaturized follicles was also increased. The presence of fibrous root sheath remnants or streamers was also observed, as a result of previous damage, localized in the dermis and hypodermis, prevalently in those patients with acute hair loss and positive pull test (Figure 2).

\section{Therapy}

Therapy was prescribed according to the severity of the disease, pull test, and trichoscopy (Table 2). In particular, clobetasol propionate $0.05 \%$ cream under occlusion was prescribed for 69 patients $(64.4 \%)$ and clobetasol propi-
Table 1. Trichoscopic features of AAI and DAA in our study.

\begin{tabular}{|c|c|c|}
\hline Trichoscopic Features & AAl, $n(\%)$ & AAD, $n(\%)$ \\
\hline $\begin{array}{l}\text { Empty yellow dots }+ \text { yellow dots with vellus hair } \\
+ \text { small hair in regrowth }+ \text { pigtail hair }\end{array}$ & $53(49.53)$ & $3(12)$ \\
\hline $\begin{array}{l}\text { Empty yellow dots }+ \text { yellow dots with vellus hair } \\
+ \text { small hair in regrowth }\end{array}$ & $29(27.10)$ & $12(48)$ \\
\hline Empty yellow dots + yellow dots with vellus hair & $2(1.86)$ & $1(4)$ \\
\hline Empty yellow dots + small hair in regrowth & $7(6.54)$ & \\
\hline $\begin{array}{l}\text { Yellow dots with vellus hair }+ \text { small hair in } \\
\text { regrowth }+ \text { pigtail hair }\end{array}$ & $4(3.73)$ & \\
\hline Yellow dots with vellus hair + pigtail hair & $3(2.80)$ & \\
\hline $\begin{array}{l}\text { Yellow dots with vellus hair }+ \text { small hair in } \\
\text { regrowth }\end{array}$ & $3(2.80)$ & \\
\hline $\begin{array}{l}\text { Empty yellow dots + yellow dots with vellus hair } \\
+ \text { pigtail hair }\end{array}$ & $2(1.86)$ & \\
\hline $\begin{array}{l}\text { Empty yellow dots + small hair in regrowth + } \\
\text { black dots }\end{array}$ & $2(1.86)$ & \\
\hline Empty yellow dots & $2(1.86)$ & \\
\hline $\begin{array}{l}\text { Empty yellow dots + yellow dots with vellus hair } \\
+ \text { dystrophic hair }\end{array}$ & & $4(16)$ \\
\hline $\begin{array}{l}\text { Empty yellow dots + yellow dots with vellus hair } \\
+ \text { pigtail hair + dystrophic hair + black dots }\end{array}$ & & $2(8)$ \\
\hline $\begin{array}{l}\text { Empty yellow dots }+ \text { yellow dots with vellus hair } \\
+ \text { black dots + dystrophic hair }\end{array}$ & & $2(8)$ \\
\hline Dystrophic hair + black dots & & $1(4)$ \\
\hline
\end{tabular}

$\mathrm{AAI}=$ alopecia areata incognita; DAA = diffuse alopecia areata.
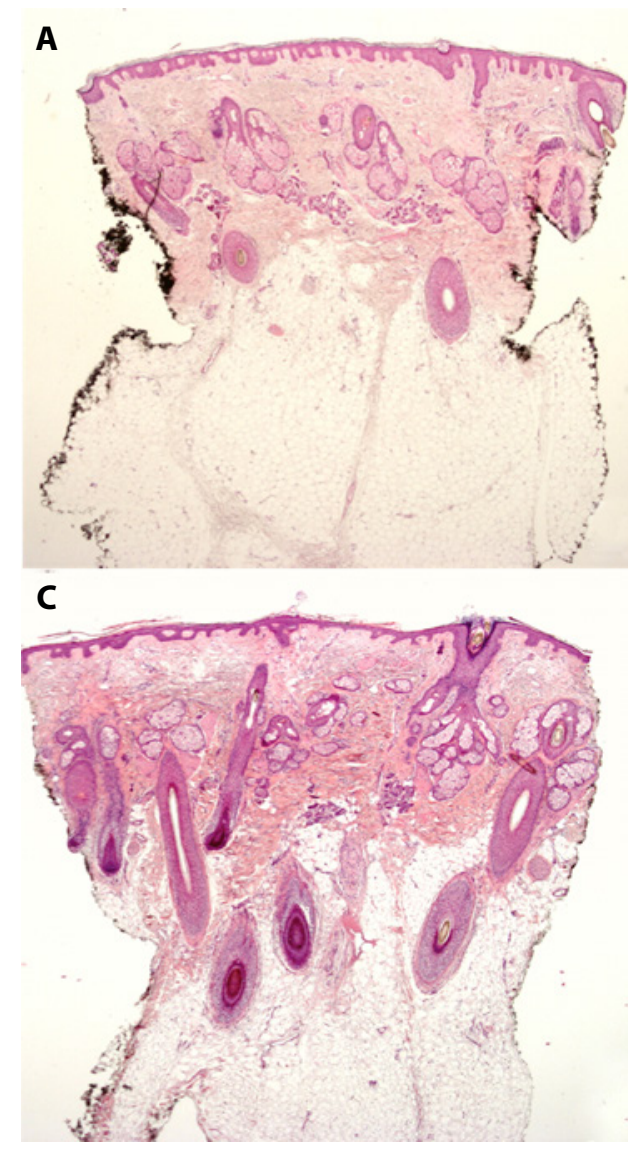

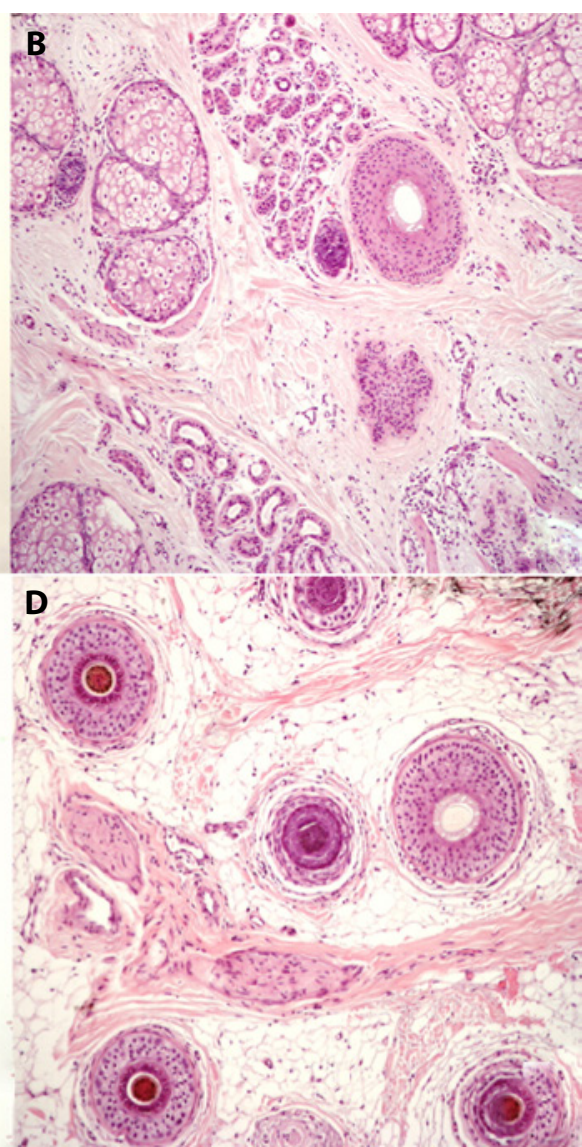

Figure 2. Histopathological section of alopecia areata incognita: $(A, B)$ reduced number of terminal anagen follicles, a dilated infundibular ostium with vellus hair and increased number of streamers (hematoxylin). Histopathological section of diffuse alopecia areata: $(\mathrm{C})$ normal number of terminal anagen follicles, slight increase in the number of streamers, an infundibular ostium dilated with cadaverized hair (H\&E, eosin, $\times 4$ ). Horizontal section: an early anagen follicle (H\&E, $\times 8)$; (D) deep perifollicular lymphocyte infiltrate (H\&E, ×8). [Copyright: (2019 Alessandrini et al.] 
Table 2. Response to topical steroid therapy in patients with AAI and DAA.

\begin{tabular}{|c|c|c|c|c|c|c|c|c|c|c|}
\hline \multirow{2}{*}{ Therapy } & \multirow{2}{*}{ n (\%) } & \multicolumn{3}{|c|}{ Stability } & \multicolumn{3}{|c|}{ Improvement } & \multicolumn{3}{|c|}{ Remission } \\
\hline & & T4 & T8 & T12 & T4 & T8 & T12 & T4 & T8 & T12 \\
\hline \multicolumn{11}{|l|}{ AAI } \\
\hline Clobetasol $0.05 \%$ cream & $\begin{array}{c}69 \\
(64.49)\end{array}$ & $\begin{array}{c}17 \\
(24.64)\end{array}$ & $\begin{array}{c}6 \\
(8.69)\end{array}$ & $\begin{array}{c}5 \\
(7.24)\end{array}$ & $\begin{array}{c}52 \\
(75.36)\end{array}$ & $\begin{array}{c}34 \\
(49.27)\end{array}$ & $\begin{array}{c}16 \\
(23.19)\end{array}$ & $\begin{array}{c}0 \\
(0)\end{array}$ & $\begin{array}{c}29 \\
(42.03)\end{array}$ & $\begin{array}{c}48 \\
(69.56)\end{array}$ \\
\hline Clobetasol $0.05 \%$ foam & $\begin{array}{c}38 \\
(35.51)\end{array}$ & $\begin{array}{c}11 \\
(28.95)\end{array}$ & $\begin{array}{c}5 \\
(13.16)\end{array}$ & $\begin{array}{c}3 \\
(7.89)\end{array}$ & $\begin{array}{c}27 \\
(71.05)\end{array}$ & $\begin{array}{c}13 \\
(34.21)\end{array}$ & $\begin{array}{c}9 \\
(23.68)\end{array}$ & $\begin{array}{c}0 \\
(0)\end{array}$ & $\begin{array}{c}20 \\
(52.63)\end{array}$ & $\begin{array}{c}26 \\
(68.42)\end{array}$ \\
\hline \multicolumn{11}{|l|}{ DAA } \\
\hline Clobetasol $0.05 \%$ cream & $\begin{array}{c}22 \\
(88)\end{array}$ & $\begin{array}{c}5 \\
(22.72)\end{array}$ & $\begin{array}{c}2 \\
(9.09)\end{array}$ & $\begin{array}{c}1 \\
(4.54)\end{array}$ & $\begin{array}{c}17 \\
(77.27)\end{array}$ & $\begin{array}{c}5 \\
(22.73)\end{array}$ & $\begin{array}{c}0 \\
(0)\end{array}$ & $\begin{array}{c}0 \\
(0)\end{array}$ & $\begin{array}{c}15 \\
(68.18)\end{array}$ & $\begin{array}{c}21 \\
(95.45)\end{array}$ \\
\hline Clobetasol $0.05 \%$ cream & $\begin{array}{c}3 \\
(12)\end{array}$ & $\begin{array}{c}1 \\
(33.33)\end{array}$ & $\begin{array}{c}0 \\
(0)\end{array}$ & $\begin{array}{c}0 \\
(0)\end{array}$ & $\begin{array}{c}2 \\
(66.66)\end{array}$ & $\begin{array}{c}1 \\
(33.33)\end{array}$ & $\begin{array}{c}1 \\
(33.33)\end{array}$ & $\begin{array}{c}0 \\
(0)\end{array}$ & $\begin{array}{c}2 \\
(66.66)\end{array}$ & $\begin{array}{c}2 \\
(66.66)\end{array}$ \\
\hline
\end{tabular}

$\mathrm{AAI}=$ alopecia areata incognita DAA $=$ diffuse alopecia areata.

onate $0.05 \%$ foam was prescribed for 38 patients $(35.5 \%)$. Topical corticosteroids were applied 3 nights/week for 4 months and then reduced in direct proportion to the patient's clinical and trichoscopic improvement, with further checks after 8 months and 1 year. In only 1 case it was necessary to prescribe intramuscular triamcinolone acetonide $40 \mathrm{mg}$ in association with clobetasol cream due to the severity of the disease. Minoxidil 2\% solution, applied twice a day, was prescribed for treating androgenetic alopecia in 88 patients $(82.2 \%)$, while in 10 patients we prescribed finasteride $2.5 \mathrm{mg} /$ day for allergic reaction. Response to therapy was assessed based on degree of severity of the disease, pull test, and trichoscopy and was rated as decrease-arrest of hair shedding and decrease-disappearance of trichoscopy signs of AAI.

Data collected showed that topical steroid therapy, although in different formulations, was very effective in AAI treatment, even just after 4 months of therapy, with results that were maintained after 8-12 months.

\section{Diffuse Alopecia Areata}

Between April 2012 and April 2017, we diagnosed DDA in 25 patients, all female $(100 \%)$ and Caucasian (25/25); average age was 38.58 years (range 24-58).

\section{Clinical Features}

All patients were characterized by diffuse hair thinning that developed quickly and was associated with increased hair loss. Only 1 patient had suffered from patchy AA in the past. Based on severity of hair thinning, they were grouped in 3 degrees per the Ludwig scale, as follows: 7 patients (28\%) with grade I, 8 patients $(32 \%)$ with grade II, and 10 patients $(40 \%)$ with grade III. Androgenetic alopecia is present in $24 \%$ of patients $(6 / 25)$.

\section{Trichoscopy}

In all patients, typical trichoscopic features were not homogeneously observed in all areas of the scalp but were seen with a higher prevalence in the parietal $(48 \%)$ and the anterior-temporal regions $(32 \%)$.

The different trichoscopic patterns were correlated with clinical presentation and severity degree: the most frequent pattern-empty yellow dots, yellow dots with vellus hair, and small hair in regrowth-corresponded to grade III (10 patients); the pattern empty yellow dots, yellow dots with vellus hair, and dystrophic hair was correlated with grade II ( 6 patients); and the remaining patterns, observed to a lesser extent, were associated with grade I (9 patients) (Figure 1; Table 1).

\section{Pathology}

The difference in pathology from AAI is a smaller number of terminal follicles in anagen, a greater number of telogen units, and numerous dilated infundibular ostia, rich in keratin and sebum. A mild inflammatory infiltrate was found around the hair follicle in the hypodermis. Hair follicles in early anagen were in greater number in DAA than in AAI. The number of miniaturized follicles was lower in DAA, according to the lower percentage of patients affected by a concomitant androgenetic alopecia. The presence of fibrous root sheath remnants or streamers was also observed, localized in the dermis and hypodermis, as a result of previous damage, prevalently in those patients with acute hair loss and positive pull test (Figure 2).

\section{Therapy}

Therapy was prescribed according to the severity of the disease, pull test, and trichoscopy. In particular, clobetasol propionate $0.05 \%$ cream under occlusion was prescribed for 22 patients $(88 \%)$ while clobetasol propionate $0.05 \%$ foam was prescribed for 3 patients (12\%). Topical corticosteroids were applied 6 nights/week for 4 months and then reduced in direct proportion to patient's clinical and trichoscopic improvement, with further checks after 8 months and 1 year. Minoxidil $2 \%$ solution twice a day 
was prescribed for treating androgenetic alopecia in 6 patients (24\%). Response to therapy was assessed based on the degree of severity of the disease, pull test, and trichoscopy.

Data collected showed that topical steroid therapy was very effective and after 1 year, almost all patients underwent complete remission of DAA (21/22) (Table 2).

\section{Discussion}

AAI cases amounted to 107, whereas the patients affected by DAA were notably fewer (only 25), pointing out a significant difference in prevalence. Both diseases were clearly prevalent in young women, with a slightly lower average age in DAA (40.5 AAI vs 38.5 DAA).

Second, androgenetic alopecia was reported in $95.23 \%$ of cases of AAI but in only $24 \%$ of cases of DAA. These data may be related to Rebora's [1] hypothesis, which affirms AAI is more frequent in patients with androgenetic alopecia because they have a high percentage of hair follicles in telogen and a low number of hair follicles in anagen with high mitotic activity. Another possible hypothesis [4] claims that not all anagen hair, if affected by an inflammatory process, undergoes mitotic arrest with formation of black dots and dystrophic hair; some follicles, in fact, could pass from anagen to telogen ("escape in telogen") without falling out immediately, thus avoiding the formation of the bald patches but instead determining a more widespread hair loss.

The role of trichoscopy in AAI was described for the first time by Tosti et al [5], who documented the presence of diffuse yellow dots in $95 \%$ of patients. Another typical trichoscopic feature of AAI is the presence of short regrowing hair (0.2-0.4 mm). Finally, in a small percentage of cases we found the presence of exclamation point hair, black dots, and dystrophic hair, confirming AAI as a variant of classic AA. According to Inui et al [6], the trichoscopic combination of yellow dots and/or short hairs in regrowth has a diagnostic sensitivity for AAI of $96 \%$.

The characteristic trichoscopic features of DAA include instead the presence of black dots and diffuse yellow dots, with an amount proportional to the severity of the disease; in this case, black dots are the result of acute damage to the follicles, not grouped to oval areas but widespread and expanded to the whole scalp. These data have never been standardized and come from our clinical experience.

The most frequent pattern seen in AAI was empty yellow dots, empty yellow dots with vellus hair, and small hair in regrowth, but the presence of pigtail hair was very frequent and almost exclusively in AAI (57.9\%), while this sign was found only in 5 patients with DDA $(20 \%)$. In our opinion, this trichoscopic sign is indicative of inflammatory damage, which causes hair to fall out and, once it regrows, prevents it from getting longer and thicker. In DAA, the follicular inflammatory insult is greater than in AAI; therefore, the trichoscopic and histopathological features are similar to those seen in "classic" patches of acute AA. This observation is reflected by the data collected by our study, which show a greater presence of dystrophic hairs and black dots in patients with AAD (9/25, 36\%) compared with patients with AAI (only 2 cases with trichoscopic evidence of black dots and no case with dystrophic hair) (Figures 3 and 4).

The most common trichoscopic sign in both diseases was the presence of empty yellow dots; $>5$ empty yellow dots in a $40 \times$ field were described in 23 patients with DAA (92\%) and in only 9 patients with AAI ( $8.4 \%$ ), corresponding to the ones with a more severe clinical presentation (grade III).

Our study pointed out also a different distribution of trichoscopy patterns in the 2 diseases: AAI predominately affects parietal-occipital regions whereas DAA manifests itself mainly in parietal and anterior-temporal regions of the scalp.

The main distinguishing histopathological characteristic between DAA and AAI is the lymphocytic infiltrate, more abundant and profound in DAA, where it is located at the level of the hypodermis and therefore represents a sign of more severe acute damage. Moreover, AAI is characterized by a preserved number of follicular units but a reduced number of terminal follicles, with numerous dilated infundibular hosts, corresponding to yellow dots [7]; an increased number of vellus hairs and miniaturized follicles is also observed, greater than androgenetic alopecia but lower than telogen effluvium [8]. In DAA the widespread hair loss is evidenced by a lower number of terminal anagen follicles, a greater number of telogen units, and numerous yellow dots. Finally, especially in patients with acute hair loss and positive pull test, there are also fibrous root sheath remnants or streamers located in the dermis and hypodermis.

The evolution of AA is unpredictable: many cases, especially those affecting less than $40 \%$ of the entire scalp, heal spontaneously but recurrences are frequent and often more severe than the onset episode [8]. In our experience, the prognosis for AAI and DDA is generally more favorable than for patchy AA and they do not tend to relapse.

No scientific papers have been published concerning therapeutic approaches and prognosis of AAI, only single-case reports [3,9]. According to our evidence, both AAI and DAA can be considered benign. In more detail, an improvement was recorded after only 4 months of therapy in $73.8 \%$ of patients with AAI and after 8 months, 49 patients $(52.4 \%)$ were already in remission. Similarly, improvement was observed in $68 \%$ of patients with DAA after 4 months of therapy, with remission in $60 \%$ of cases after 8 months. High-potency topical steroids are therefore the therapy of choice for these forms of nonpatchy AA. 


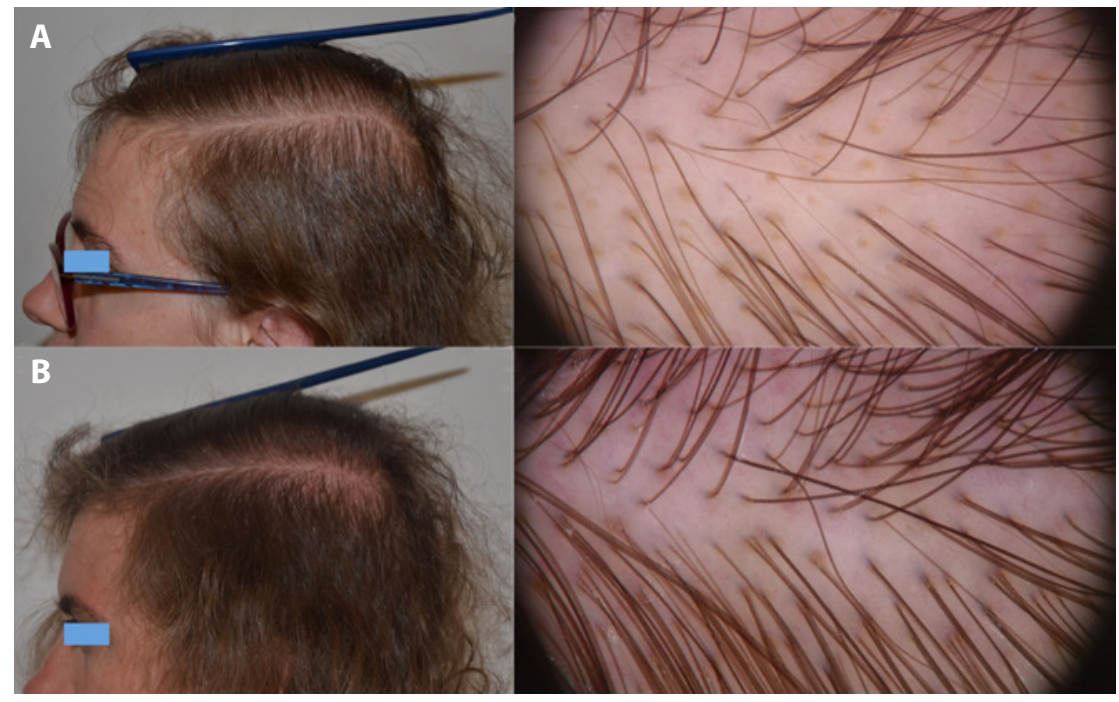

Figure 3. Clinical and trichoscopic improvement in a patient with alopecia areata incognita: (A) at the first visit; (B) after 1 year of therapy. [Copyright: (O2019 Alessandrini et al.]

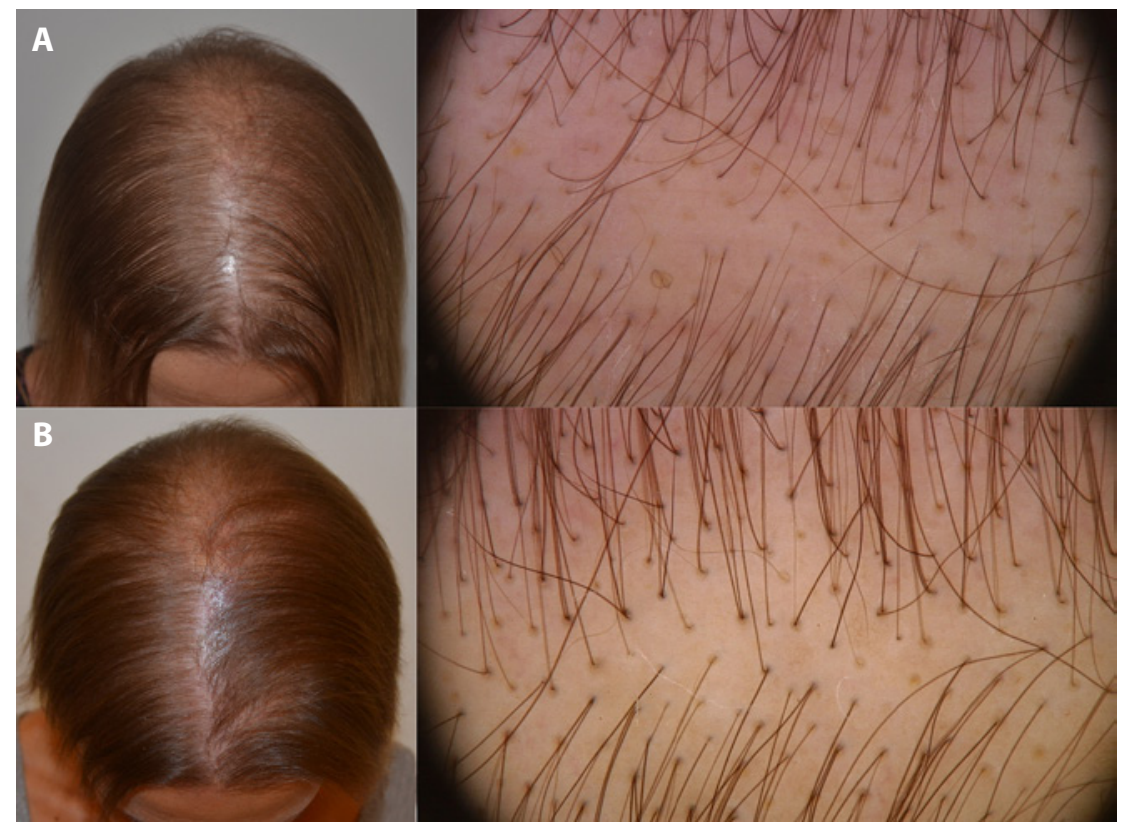

Figure 4. Clinical and trichoscopic improvement of a patient with diffuse alopecia areata: (A) at the first visit; (B) after 4 months of therapy. [Copyright: @2019 Alessandrini et al.]

\section{Conclusions}

In our experience, both AAI and DAA are typical of young women with associated androgenetic alopecia, and they present with diffuse hair thinning. However, from our analysis, we found that DAA had a greater involvement of the parietal and anterior-temporal regions, whereas AAI manifests itself mainly in the occipital and parietal regions. The clinical examination alone is insuffi- cient. Trichoscopy is a noninvasive diagnostic technique that directly evaluates the scalp and assumes enormous importance in the diagnosis and follow-up of hair diseases. The most frequent pattern seen in our series of patients with AAI was empty yellow dots, yellow dots with vellus hair, and small hair in regrowth, but the presence of pigtail hair was very frequent and almost exclusive in AAI; thus, this trichoscopic sign can represent a useful clue in the differential diagnosis between the 2 pathologies. Moreover, in cases of DAA, the finding of dystrophic hair and black dots is more frequent, indicating the acute and deep inflammatory damage present in this disease. Another important role of trichoscopy is to select the best site for biopsy, in order to correlate trichoscopy with the histopathological findings. Both AAI and DAA have a benign course and are responsive to topical steroid therapy.

In conclusion, in a case of a patient with excessive hair loss in a short time in whom trichoscopy reveals typical signs of AA in a diffuse location, a suspicion of AAI or DAA must be considered along with histological examination.

\section{References}

1. Rebora A. Alopecia areata incognita: a hypothesis. Dermatologica. 1987;174(5): 214-218.

2. Molina L, Donati A, Valente NS, et al. Alopecia areata incognita. Clinics (Sao Paulo). 2011;66(3):513-515.

3. Ludwig E. Classification of the types of androgenetic alopecia (common baldness) occurring in the female sex. Br J Dermatol. 1977;97(3):247-254

4. Park J, Song KH, Nam KH. Circumscribed alopecia areata incognita. Australas J Dermatol. 2013;54(1):52-54.

5. Tosti A, Whiting D, Iorizzo M, et al. The role of scalp dermoscopy in the diagnosis of alopecia areata incognita. J Am Acad Dermatol. 2008;59(1):64-67.

6. Inui S, Nakajima T, Itami S. Significance of dermoscopy in acute diffuse and total alopecia of the female scalp: review of twenty cases. Dermatology. 2008;217(4):333336.

7. Müller CSL, El Shabrawi-Caelen L. "Follicular Swiss cheese" pattern-another histopathologic clue to alopecia areata. $J$ Cutan Pathol. 2011;38(2):185-189.

8. Miteva M, Misciali C, Fanti PA, et al. Histopathologic features of alopecia areata incognito: a review of 46 cases. J Cutan Pathol. 2012;39(6):596-602.

9. Strazzulla LC, Wang EHC, Avila L, et al. Alopecia areata: an appraisal of new treatment approaches and overview of current therapies. J Am Acad Dermatol. 2018;78(1):15-24. 